\title{
Range Estimation in Multicarrier Systems in the Presence of Interference: Performance Limits and Optimal Signal Design
}

\author{
Yasir Karisan, Student Member, IEEE, Davide Dardari, Senior Member, IEEE, \\ Sinan Gezici, Senior Member, IEEE, Antonio A. D'Amico, and Umberto Mengali, Life Fellow, IEEE
}

\begin{abstract}
Theoretical limits on time-of-arrival (equivalently, range) estimation are derived for multicarrier systems in the presence of interference. Specifically, closed-form expressions are obtained for Cramer-Rao bounds (CRBs) in various scenarios. In addition, based on CRB expressions, an optimal power allocation (or, spectrum shaping) strategy is proposed. This strategy considers the constraints not only from the sensed interference level but also from the regulatory emission mask. Numerical results are presented to illustrate the improvements achievable with the optimal power allocation scheme, and a maximum likelihood time-of-arrival estimation algorithm is studied to assess the effects of the proposed approach in practical estimators.
\end{abstract}

Index Terms-Ranging, time-of-arrival (TOA) estimation, interference, orthogonal frequency division multiplexing (OFDM), Cramer-Rao bound (CRB), cognitive radio.

\section{INTRODUCTION}

A CCURATE position estimation in cellular networks can facilitate various applications and services such as enhanced-911, improved fraud detection, location sensitive billing, intelligent transport systems, and improved traffic management [1]. Also, for short-range networks, position estimation can enable applications such as inventory tracking, intruder detection, tracking of fire-fighters and miners, home automation and patient monitoring [3], [4]. Commonly, position estimation is performed in two steps [5]. In the first step, position related signal parameters, such as time-of arrival (TOA), angle-of-arrival (AOA), and received signal strength (RSS), are estimated. Then, in the second step, the position is estimated based on the signal parameters obtained in the first step. Fingerprinting approaches or statistical techniques can be used in the second step, depending on the accuracy requirements and system constraints [5]. In order to improve

Manuscript received October 18, 2010; revised February 24 and May 31, 2011; accepted June 21, 2011. The associate editor coordinating the review of this paper and approving it for publication was M. Shafi.

Y. Karisan is with the Department of Electrical and Computer Engineering, The Ohio State University, 205 Dreese Laboratories, 2015 Neil Avenue Columbus, OH 43210, USA (e-mail: yasirkarisan@gmail.com).

D. Dardari is with WiLAB-DEIS, University of Bologna at Cesena, via Venezia 52, 47023 Cesena (FC), Italy (e-mail: ddardari@ieee.org).

S. Gezici is with the Department of Electrical and Electronics Engineering, Bilkent University, Bilkent, Ankara 06800, Turkey (e-mail: gezici@ee.bilkent.edu.tr).

A. A. D'Amico and U. Mengali are with the Department of Information Engineering of the University of Pisa, Via Caruso 16, Pisa, Italy (e-mail: \{antonio.damico, umberto.mengali\}@iet.unipi.it.).

This research was supported in part by the European Commission in the framework of the FP7 Network of Excellence in Wireless COMmunications NEWCOM++ (contract no. 216715), in part by the FP7 European Project EUWB (Grant no. 215669), and in part by the WiMAGIC project of the EC Seventh Framework Programme (FP7) under grant agreement no. 215167. Part of this research was presented in the IEEE International Conference on Communications (ICC) Workshops, Dresden, Germany, June 2009.

Digital Object Identifier 10.1109/TWC.2011.072511.101844 positioning accuracy, position related parameters in the first step should be estimated as accurately as possible. Since the RSS parameter cannot provide accurate position information and the AOA parameter commonly requires the use of multiple antennas, the TOA parameter is usually employed for obtaining accurate position information in wireless systems [6]-[10]. The focus of this paper is on TOA (equivalently, range) estimation in multicarrier systems.

Multicarrier signaling and, specifically, orthogonal frequency division multiplexing (OFDM), is a suitable technique for high data rate systems due to its unique characteristics, such as robustness against inter-symbol interference (ISI) and implementation simplicity provided by efficient fast Fourier transform (FFT) algorithms. For these reasons it is considered for a variety of applications such as wireless local area networks, digital audio/video broadcasting, asymmetric digital subscriber lines (ADSLs), 3GPP-LTE, and the IEEE 802.16 WiMAX standard [11]-[13]. Multicarrier signals can also be employed for TOA estimation for positioning purposes [14]-[17]. In [14], OFDM is studied for time-based range estimation, and Cramer-Rao bounds (CRBs) and maximumlikelihood (ML) estimators are derived. In [15] a positioning system is investigated based on super-resolution TOA estimation in OFDM systems for indoor environments and the performance of various super-resolution TOA estimators is compared via simulations. Also, positioning using both OFDM based communications and the global navigation satellite system (GNSS) is studied in [17], where time-difference-ofarrival (TDOA) information from an OFDM system is utilized.

Since multicarrier systems have inherent frequency diversity, it is crucial to exploit such a diversity for TOA (range) estimation. In [18], the theoretical limits on range estimation are studied for dispersed spectrum cognitive radio (CR) systems, which use a number of frequency bands in the spectrum. A receiver with multiple branches is considered, where each branch processes a narrowband signal at a different center frequency, and the CRBs on range estimation are obtained. In [19] the same problem is considered and practical two-step range estimation algorithms are proposed. It is observed that the frequency diversity in the system can be utilized for range estimation. The same goal can be pursued exploiting space diversity, as discussed in [20].

Although range estimation has been investigated for multicarrier systems, no studies have considered range estimation for multicarrier systems in the presence of interference nor have CRB expressions been obtained for range estimation accuracy in that case. In addition, although it is known that the coefficients of the subcarriers can be adjusted in various ways to provide spectrum agility or capacity improvements [21], the 
optimal power allocation for subcarriers for range accuracy enhancement has not been investigated in the presence of fading and interference.

In this paper CRB expressions are derived for range estimation in the presence of interference and an optimal power allocation (or, spectrum shaping) strategy is proposed. Although [25] deals with a related issue and focuses on the problem of sampling clock frequency mismatch between a transmitter and a receiver in an OFDM system, the CRB expressions obtained in that study are concerned with the estimation of clock frequency offset between the transmitter and the receiver, and it is assumed that no interference exists in the system. Channel delay estimation, and implicitly TOA estimation, is also addressed in [26] in the context of multiple-input multiple-output OFDM systems. However, the main issue there is to separate signals arriving from different transmitting antennas.

An additional contribution of our study is the development of an optimal power allocation strategy for minimizing the $\mathrm{CRB}$ on time delay estimation under practical constraints (such as the regulatory limits on the power spectral density), and the investigation of an ML TOA estimation algorithm in order to assess the effects of the optimal power allocation algorithm in practical systems. Numerical results and simulation examples are provided to compare conventional and optimal power allocation strategies.

In summary the main contributions of our investigation are:

- A closed-form expression of the CRB for range estimation in multicarrier systems in the presence of interference.

- An optimal power allocation (or, spectrum shaping) strategy based on the CRB expressions.

- The derivation of the ML TOA estimator and the assessment of the optimal spectrum shaping on the estimator performance.

It is worth noting that the problem formulation and its solution can be viewed as the counterparts of the capacity maximization problem and the corresponding water-filling algorithm [21], [27]. Also, the results of this study can be exploited for positioning systems employing detection and avoidance (DAA) techniques, perhaps working under the CR paradigm, since interference awareness and feedback from receiver to transmitter are considered in the theoretical analysis [22]-[24]. Another motivation for this study is that the range estimation accuracy of practical multicarrier systems can be improved via the proposed approaches since both interference and regulatory constraints are taken into account in the analysis. Since multicarrier signaling has recently been employed in many systems, and positioning applications are becoming more and more popular, accurate range estimation in practical multicarrier systems is of significant importance.

It should also be emphasized that the difference of the CRB expressions for multicarrier systems in this work from the other studies in the literature is mainly in the presence of interference. As discussed in Section IV, the performance limits on TOA estimation strongly depend not only on the interference power but also on the strategy adopted to counteract it. In particular it is shown that the frequency diversity can be efficiently exploited using all the signal sub-carriers rather than only those without interference.

The remainder of the paper is organized as follows. In Section II a multicarrier signal model is introduced. Various
CRBs for TOA estimation are derived in Section III for general and special cases. In Section III-C the CRB expressions are exploited to formulate a power allocation strategy that maximizes the range estimation accuracy. Performance evaluations are presented in Section IV and concluding remarks are made in Section V.

\section{Signal Model}

Thanks to their flexibility in utilizing the radio spectrum, multicarrier signals are commonly employed in communication systems. In this paper a multicarrier signaling scheme is adopted and the transmitted baseband signal is modeled as ${ }^{1}$

$$
s(t)=\sum_{k=1}^{K} \sqrt{w_{k}} p(t) e^{\jmath 2 \pi f_{k} t},
$$

over the symbol interval $\left[-T_{s} / 2, T_{s} / 2\right]$. In this equation $f_{k}=$ $(k-K / 2) \Delta$ is the $k$ th subcarrier frequency shift with respect to the center frequency, $\Delta$ is the subcarrier spacing, and $p(t)$ is a pulse with duration $T_{s}$ and energy $E_{p}$. The weights $w_{k} \geq 0$ permit spectrum shaping and

$$
P_{t}=\frac{E_{p}}{T_{s}} \sum_{k=1}^{K} w_{k}
$$

represents the power of the baseband signal. ${ }^{2}$ In practice, the weights $w_{k}$ are limited by peak power constraints, as is detailed in Section III-C when considering the optimal signal spectrum.

Assuming that $\Delta$ is small compared to the channel coherence bandwidth, so that each subcarrier experiences locally flat fading [27], it can be shown that the baseband received signal corresponding to (1) is

$$
r(t) \cong s_{r}(t-\tau)+z(t)
$$

with

$$
s_{r}(t)=\sum_{k=1}^{K} \alpha_{k} \sqrt{w_{k}} p(t) e^{\jmath 2 \pi f_{k} t},
$$

where $\tau$ is the propagation delay, $\alpha_{k}=a_{k} e^{\jmath \phi_{k}}$ denotes the complex channel coefficient at frequency $f_{k}$, and $z(t)$ is the total disturbance due to thermal noise and interference. In particular, $z(t)$ is the sum of two terms, say $z_{N}(t)$ and $z_{I}(t)$, where $z_{N}(t)$ is complex additive white Gaussian noise (AWGN) with spectral density $N_{0}$ for each component, and $z_{I}(t)$ is a stationary interference term with power spectral density $S_{I}(f)$ for each component. Thus, the power spectral density of each component of $z(t)$ is $S_{z}(f)=N_{0}+S_{I}(f)$. In addition, the interference is modeled as a zero-mean complex Gaussian process. The approximation of interference by a zero-mean complex Gaussian process is justified in [34] for the case in which the interferers also employ a multicarrier signaling scheme.

It should be noted that the received signal model in (3)(4) applies to a multipath channel, and the propagation delay $\tau$ approximately represents the delay of the shortest path. ${ }^{3}$

\footnotetext{
${ }^{1} \mathrm{~A}$ guard interval between symbols is assumed to avoid inter-symbol interference at the receiver.

${ }^{2}$ The corresponding RF power is $\frac{E_{p}}{2 T_{s}} \sum_{k=1}^{K} w_{k}$.

${ }^{3}$ This approximation is quite accurate in the presence of line-of-sight propagation.
} 
For example, with line-of-sight propagation $\tau$ coincides with the delay of the direct path and, under such conditions, $\tau$ is related to the range (distance) between the transmitter and the receiver. Based on a number of range estimates between a terminal and a number of reference devices, the position of the terminal can be estimated [5].

\section{CRBs on TOA Estimation In THE PRESENCE OF INTERFERENCE}

In this section we consider the best achievable accuracy in estimating the TOA parameter $\tau$ from the observation of $r(t)$ in the presence of interference. ${ }^{4}$

The Fourier transform of $s_{r}(t-\tau)$ in (4) is

$$
S_{r}(f, \boldsymbol{\theta})=\sum_{k=1}^{K} \alpha_{k} \sqrt{w_{k}} P\left(f-f_{k}\right) e^{-\jmath 2 \pi f \tau},
$$

where $P(f)$ is the Fourier transform of $p(t)$, and $\boldsymbol{\theta} \triangleq$ $\left[\tau a_{1} \cdots a_{K} \phi_{1} \cdots \phi_{K}\right]$ is a vector collecting all the channel parameters. In computing the CRB for the estimation of $\tau$, two different approaches can be adopted. In one case, called joint bounding, the estimation process concerns all the components of $\boldsymbol{\theta}$ and a bound is derived for each of them. In the other case the focus is on $\tau$ alone and the other components of $\boldsymbol{\theta}$ are regarded as known parameters. This is referred to as conditional bounding [30].

\section{A. Joint Bounding}

As the disturbance $z(t)$ is colored, we assume without loss of generality that the received signal is first passed through a whitening filter with a frequency response [31]

$$
|H(f)|^{2}=\frac{1}{S_{z}(f)} .
$$

Accordingly, the log-likelihood function can be written as ${ }^{5}$

$$
\ln \Lambda(\widetilde{\boldsymbol{\theta}})=\Re\left\{\int_{-\infty}^{\infty} x(t) u^{*}(t, \widetilde{\boldsymbol{\theta}}) d t\right\}-\frac{1}{2} \int_{-\infty}^{\infty}|u(t, \widetilde{\boldsymbol{\theta}})|^{2} d t
$$

where $\widetilde{\boldsymbol{\theta}}$ is a possible value of $\boldsymbol{\theta}, x(t)=r(t) \otimes h(t)$ is the convolution of the received waveform $r(t)$ with the impulse response of the whitening filter $h(t), u(t, \widetilde{\boldsymbol{\theta}})=\tilde{s}_{r}(t-\tilde{\tau}) \otimes h(t)$, and

$$
\tilde{s}_{r}(t)=\sum_{k=1}^{K} \tilde{\alpha}_{k} \sqrt{w_{k}} p(t) e^{j 2 \pi f_{k} t} .
$$

The derivation of (7) is presented in Appendix A.

Equivalently, the whitening operation can be performed by correlating $r(t)$ with a pulse $g(t, \widetilde{\boldsymbol{\theta}})$ with the following Fourier transform [31]

$$
G(f, \widetilde{\boldsymbol{\theta}}) \propto \tilde{S}_{r}(f, \widetilde{\boldsymbol{\theta}}) / S_{z}(f)
$$

and the log-likelihood function is obtained as [31]

$$
\begin{aligned}
\ln \Lambda(\widetilde{\boldsymbol{\theta}}) & =\Re\left\{\int_{-\infty}^{\infty} r(t) g^{*}(t, \widetilde{\boldsymbol{\theta}}) d t\right\} \\
& -\frac{1}{2} \int_{-\infty}^{\infty} \tilde{s}_{r}(t-\tilde{\tau}) g^{*}(t, \widetilde{\boldsymbol{\theta}}) d t
\end{aligned}
$$

\footnotetext{
${ }^{4}$ The observation interval is assumed sufficiently long so as to comprise the whole received signal notwithstanding the a priori uncertainty on the actual value of $\tau$.

${ }^{5} \Re\{x\}$ and $\Im\{x\}$ denote the real and the imaginary parts of $x$, respectively.
}

The derivation of (10) is presented in Appendix B.

The CRB for TOA estimation is computed as

$$
\operatorname{Var}(\hat{\tau}) \geq\left[\mathbf{J}^{-1}\right]_{1,1}=\mathrm{CRB},
$$

where $\mathbf{J}$ is the Fisher information matrix (FIM) with elements [31], [33]

$$
[\mathbf{J}]_{m, n}=\Re\left\{\int_{-\infty}^{\infty} \frac{\partial \tilde{S}_{r}^{*}(f, \boldsymbol{\theta})}{\partial \tilde{\theta}_{m}} S_{z}^{-1}(f) \frac{\partial \tilde{S}_{r}(f, \boldsymbol{\theta})}{\partial \tilde{\theta}_{n}} d f\right\} .
$$

In (12), $\tilde{\theta}_{n}$ is the $n$th element of $\widetilde{\boldsymbol{\theta}}$ and, with a slight abuse of notation, $\partial \tilde{S}_{r}(f, \boldsymbol{\theta}) / \partial \tilde{\theta}_{n}$ denotes the partial derivative of $\tilde{S}_{r}(f, \widetilde{\boldsymbol{\theta}})$ with respect to $\tilde{\theta}_{n}$ computed for $\widetilde{\boldsymbol{\theta}}=\boldsymbol{\theta}$.

After some manipulations from (5) and (12) it is found that ${ }^{6}$

$$
\mathbf{J}=\left[\begin{array}{ccc}
\mathrm{J}_{\tau \tau} & \mathbf{J}_{\tau a} & \mathbf{J}_{\tau \phi} \\
\mathbf{J}_{\tau a}^{T} & \mathbf{J}_{a a} & \mathbf{J}_{a \phi} \\
\mathbf{J}_{\tau \phi}^{T} & \mathbf{J}_{a \phi}^{T} & \mathbf{J}_{\phi \phi}
\end{array}\right]
$$

where the elements of $\mathbf{J}$ are expressed as follows:

$$
\begin{gathered}
\mathrm{J}_{\tau \tau}=4 \pi^{2} \Re\left\{\sum_{k=1}^{K} \sum_{l=1}^{K} \alpha_{k}^{*} \alpha_{l} \sqrt{w_{k} w_{l}} y_{k, l}(2)\right\} \\
{\left[\mathbf{J}_{\tau a}\right]_{m}=-2 \pi \sqrt{w_{m}} \Im\left\{e^{j \phi_{m}} \sum_{k=1}^{K} \alpha_{k}^{*} \sqrt{w_{k}} y_{k, m}(1)\right\},} \\
{\left[\mathbf{J}_{\tau \phi}\right]_{m}=-2 \pi \sqrt{w_{m}} \Re\left\{\alpha_{m} \sum_{k=1}^{K} \alpha_{k}^{*} \sqrt{w_{k}} y_{k, m}(1)\right\}} \\
{\left[\mathbf{J}_{a a}\right]_{m, n}=\sqrt{w_{m} w_{n}} \Re\left\{e^{j\left(\phi_{n}-\phi_{m}\right)} y_{m, n}(0)\right\}} \\
{\left[\mathbf{J}_{a \phi}\right]_{m, n}=-\sqrt{w_{m} w_{n}} \Im\left\{e^{-j \phi_{m}} \alpha_{n} y_{m, n}(0)\right\}} \\
{\left[\mathbf{J}_{\phi \phi}\right]_{m, n}=\sqrt{w_{m} w_{n}} \Re\left\{\alpha_{m}^{*} \alpha_{n} y_{m, n}(0)\right\}}
\end{gathered}
$$

with

$$
y_{m, n}(i) \triangleq \int_{-\infty}^{\infty} f^{i} S_{z}^{-1}(f) P^{*}\left(f-f_{m}\right) P\left(f-f_{n}\right) d f
$$

for $i=0,1,2$ and $m, n=1,2, \ldots, K$.

Inspection of (13) reveals that the FIM can be put in the form of

$$
\mathbf{J}=\left[\begin{array}{ll}
\mathrm{J}_{\tau \tau} & \mathbf{B} \\
\mathbf{B}^{T} & \mathbf{C}
\end{array}\right]
$$

with $\mathbf{B} \triangleq\left[\begin{array}{ll}\mathbf{J}_{\tau a} & \mathbf{J}_{\tau \phi}\end{array}\right]$ and $\mathbf{C} \triangleq\left[\begin{array}{cc}\mathbf{J}_{a a} & \mathbf{J}_{a \phi} \\ \mathbf{J}_{a \phi}^{T} & \mathbf{J}_{\phi \phi}\end{array}\right]$. Thus, substituting (21) into (11) yields

$$
\mathrm{CRB}=\left(\mathrm{J}_{\tau \tau}-\mathbf{B C}^{-1} \mathbf{B}^{T}\right)^{-1} .
$$

Equation (22) takes simpler forms in the following special cases.

\footnotetext{
${ }^{6} \mathbf{A}^{T}$ is the transpose of $\mathbf{A}$.
} 
1) Disjoint Spectra: If $|P(f)|$ is approximately zero outside $-\Delta / 2 \leq f \leq \Delta / 2$, from (20) we have $y_{m, n}(i)=0$ for $m \neq n$ and (14) $-(19)$ become

$$
\begin{aligned}
& \mathrm{J}_{\tau \tau}=4 \pi^{2} \sum_{k=1}^{K}\left|\alpha_{k}\right|^{2} w_{k} \eta_{k}(2), \\
& \mathbf{J}_{\tau a}=\mathbf{0}, \\
& {\left[\mathbf{J}_{\tau \phi}\right]_{m}=-2 \pi w_{m}\left|\alpha_{m}\right|^{2} \eta_{m}(1),} \\
& \mathbf{J}_{a a}=\operatorname{diag}\left\{w_{1} \eta_{1}(0), w_{2} \eta_{2}(0), \ldots, w_{K} \eta_{K}(0)\right\}, \\
& \mathbf{J}_{a \phi}=\mathbf{0}, \\
& \mathbf{J}_{\phi \phi}=\operatorname{diag}\left\{w_{1}\left|\alpha_{1}\right|^{2} \eta_{1}(0), \ldots, w_{K}\left|\alpha_{K}\right|^{2} \eta_{K}(0)\right\},
\end{aligned}
$$

with

$$
\eta_{k}(i) \triangleq \int_{-\infty}^{\infty} f^{i} S_{z}^{-1}(f)\left|P\left(f-f_{k}\right)\right|^{2} d f, \quad i=0,1,2 .
$$

Thus, substituting (23)-(28) into (22) yields

$$
\mathrm{CRB}=\left(\mathrm{J}_{\tau \tau}-\mathbf{J}_{\tau \phi} \mathbf{J}_{\phi \phi}^{-1} \mathbf{J}_{\tau \phi}^{T}\right)^{-1}=\left(\sum_{k=1}^{K} w_{k} \lambda_{k}\right)^{-1},
$$

with

$$
\lambda_{k}=4 \pi^{2}\left|\alpha_{k}\right|^{2}\left(\eta_{k}(2)-\frac{\eta_{k}^{2}(1)}{\eta_{k}(0)}\right) .
$$

We see that the contribution of each subcarrier to the CRB is determined by the corresponding weight $w_{k}$, the squared channel gain $\left|\alpha_{k}\right|^{2}$, the spectrum of pulse $p(t)$, and the power spectral density $S_{I}(f)$ of the interference around $f_{k}$. It should be noted that expression (31) does not require any alignment between the interferer center frequency and the subcarrier frequencies.

2) Slowly-varying $S_{z}(f)$ : The coefficient $\lambda_{k}$ in (31) can be further simplified assuming $S_{z}(f) \cong S_{z}\left(f_{k}\right)=N_{0}+S_{I}\left(f_{k}\right)$ for $\left|f-f_{k}\right| \leq \Delta / 2 \forall k$. Correspondingly (29) becomes

$$
\begin{aligned}
\eta_{k}(i) & \cong \frac{1}{S_{z}\left(f_{k}\right)} \int_{-\infty}^{\infty} f^{i}\left|P\left(f-f_{k}\right)\right|^{2} d f \\
& =\frac{1}{S_{z}\left(f_{k}\right)} \int_{-\infty}^{\infty}\left(f+f_{k}\right)^{i}|P(f)|^{2} d f .
\end{aligned}
$$

Then, defining

$$
\beta_{i} \triangleq \frac{1}{E_{p}} \int_{-\infty}^{\infty} f^{i}|P(f)|^{2} d f \quad i=1,2
$$

and bearing in mind that

$$
\int_{-\infty}^{\infty}|P(f)|^{2} d f=E_{p}
$$

we obtain

$$
\begin{gathered}
\eta_{k}(2)=\frac{E_{p}}{S_{z}\left(f_{k}\right)}\left(\beta_{2}+2 f_{k} \beta_{1}+f_{k}^{2}\right), \\
\eta_{k}(1)=\frac{E_{p}}{S_{z}\left(f_{k}\right)}\left(\beta_{1}+f_{k}\right), \\
\eta_{k}(0)=\frac{E_{p}}{S_{z}\left(f_{k}\right)} .
\end{gathered}
$$

Finally, substituting (35)-(37) into (31) produces

$$
\lambda_{k}=\frac{4 \pi^{2} E_{p}\left|\alpha_{k}\right|^{2}\left(\beta_{2}-\beta_{1}^{2}\right)}{N_{0}+S_{I}\left(f_{k}\right)} .
$$

The physical meanings of $\beta_{2}$ and $\beta_{1}$ are as follows. From (33) we recognize that $\beta_{2}$ gives the mean-squared bandwidth of $p(t)$ while $\beta_{1}$ represents the skewness of the spectrum $|P(f)|^{2}$. Note that, if $p(t)$ is real valued, $|P(f)|$ is an even function and $\beta_{1}$ is zero. In that case, $\lambda_{k}$ is proportional to the multiplication of the mean-squared bandwidth and the signal-to-interference-plus-noise ratio (SINR) at subcarrier $k$. On the other hand, $\eta_{k}(i)$ in (32) represents the $i$ th moment of the pulse spectrum $P(f)$ normalized to the spectrum of the interference.

Equation (38) indicates that the contribution of the $k$ th subcarrier is proportional to $\left|\alpha_{k}\right|^{2} /\left(N_{0}+S_{I}\left(f_{k}\right)\right)$. Thus, $\lambda_{k}$ gets larger and the CRB reduces as the channel gain increases and/or the interference spectral density around $f_{k}$ decreases.

The use of $\lambda_{k}$ 's in (38) is based inherently on the condition that the subcarrier frequency bands align exactly with the boundaries of the frequency ranges over which the interference spectral density $S_{I}(f)$ can be approximated by a constant. In case this condition is violated, (31) can still be resorted to find $\lambda_{k}$ 's, but this approach involves more computational complexity. Note that the assumption of a slowly-varying $S_{z}(f)$ serves only to simplify the computation of the coefficients $\lambda_{k}$. If the assumption does not hold, equation (30) is still valid but the $\lambda_{k}$ must be derived from (29) and (31) with some extra effort.

\section{B. Conditional Bounding}

Assuming that the components of $\boldsymbol{\theta}$ are all known except for $\tau$, the CRB for TOA estimation can be derived from (11)(12) by considering the estimation of a single parameter. As a result we get

$$
\mathrm{CRB}=\int_{-\infty}^{\infty}\left|\frac{\partial \tilde{S}_{r}(f, \boldsymbol{\theta})}{\partial \tilde{\tau}}\right|^{2} S_{z}^{-1}(f) d f=\left[\mathrm{J}_{\tau \tau}\right]^{-1},
$$

where $\mathrm{J}_{\tau \tau}$ is still as in (14). Comparison with (30) reveals that the conditional bound is equal or less than the joint bound. This is intuitively clear because precise information on parameters $\left[a_{1} \cdots a_{K} \phi_{1} \cdots \phi_{K}\right]$ is assumed in (39).

1) Disjoint Spectra and Slowly-varying $S_{z}(f)$ : In this case, $\mathrm{J}_{\tau \tau}$ and $\eta_{k}(2)$ are given by (23) and (35), respectively. Thus, the CRB takes the same form as in the joint bounding case (c.f. (30)):

$$
\mathrm{CRB}=\left(\sum_{k=1}^{K} w_{k} \bar{\lambda}_{k}\right)^{-1}
$$

with

$$
\bar{\lambda}_{k} \triangleq \frac{4 \pi^{2} E_{p}\left|\alpha_{k}\right|^{2}\left(\beta_{2}+2 f_{k} \beta_{1}+f_{k}^{2}\right)}{N_{0}+S_{I}\left(f_{k}\right)} .
$$

Note that the difference

$$
\bar{\lambda}_{k}-\lambda_{k}=\frac{4 \pi^{2} E_{p}\left|\alpha_{k}\right|^{2}\left(\beta_{1}+f_{k}\right)^{2}}{N_{0}+S_{I}\left(f_{k}\right)}
$$

is positive so that $\bar{\lambda}_{k}>\lambda_{k}$. This agrees with our intuition that conditional bounding gives a lower CRB than joint bounding. 


\section{Optimal Weights}

Now we concentrate on the weight assignment that minimizes the CRB. It is assumed that the interference spectral density $S_{I}(f)$ is known or estimated, which is commonly possible in a system employing DAA or, more generally, CR techniques [22], [23], [28], [29]. In Section IV, the effects of uncertainties in the knowledge of $S_{I}(f)$ will be investigated. The optimal weights must satisfy constraints on the emitted signal spectrum imposed by regulatory masks (for example, the FCC mask for ultra-wide bandwidth signals [35]). Let $B(f)$ denote the equivalent baseband version of the power spectral density mask. Then, defining $\boldsymbol{w} \triangleq$ $\left(w_{1}, w_{2}, \ldots, w_{K}\right)^{T}$ and $\boldsymbol{\lambda} \triangleq\left(\lambda_{1}, \lambda_{2}, \ldots \lambda_{K}\right)^{T}$ (c.f. (30) and (40)), the optimal weights are found as the solution of the following problem:

$$
\underset{\boldsymbol{w}}{\operatorname{maximize}} \lambda^{T} \boldsymbol{w}
$$

subject to

$$
\begin{array}{r}
\mathbf{1}^{T} \boldsymbol{w} \leq 1 \\
\boldsymbol{w} \geq \mathbf{0} \\
\boldsymbol{w} \leq \boldsymbol{b}
\end{array}
$$

where $\boldsymbol{x} \leq \boldsymbol{y}$ means that each element of $\boldsymbol{x}$ is smaller than or equal to the corresponding element of $\boldsymbol{y}, \mathbf{1}$ is the vector of all ones, $\boldsymbol{b} \triangleq\left[\begin{array}{llll}b_{1} & b_{2} & \cdots & b_{K}\end{array}\right]^{T}$, and $b_{k} \triangleq B\left(f_{k}\right) \Delta / P_{t}$ is the normalized emission power constraint on the $k$ th subcarrier.

This is a classical linear programming problem and its solution can be obtained in closed-form as follows: Without loss of generality assume that the $\lambda_{k}$ are in a decreasing order, ${ }^{7}$ i.e., $\lambda_{1}>\lambda_{2}>\cdots>\lambda_{K}$. Then, the optimal weights are recursively computed as

$$
w_{i}^{(\mathrm{opt})}=\min \left\{b_{i}, 1-\sum_{j=1}^{i-1} w_{j}^{(\mathrm{opt})}\right\},
$$

for $i=2,3, \ldots, K$, with $w_{1}^{(\mathrm{opt})}=\min \left\{1, b_{1}\right\}$. The derivation of (47) is presented in Appendix C.

An alternative way of writing (47) is

$$
\begin{aligned}
& w_{1}^{(\mathrm{opt})}=\min \left\{1, b_{1}\right\}, \\
& w_{2}^{(\mathrm{opt})}=\min \left\{1-w_{1}^{(\mathrm{opt})}, b_{2}\right\}, \\
& w_{3}^{(\mathrm{opt})}=\min \left\{1-w_{1}^{(\mathrm{opt})}-w_{2}^{(\mathrm{opt})}, b_{3}\right\},
\end{aligned}
$$

and so on. This result has the following intuitive interpretation. We start by selecting the best subcarrier (the one associated to the largest component of $\boldsymbol{\lambda}$ ) and we assign to it the maximum allowed power, which is $\min \left\{1, b_{1}\right\}$. Next, we select the best of the remaining subcarriers and again assign to it the maximum allowed power (which is the minimum between $b_{2}$ and the residual power $\left.1-w_{1}^{(\mathrm{opt})}\right)$. We proceed in this way until all the available power is used or no other subcarriers are available (which happens if $\sum_{i=1}^{K} b_{i} \leq 1$ ).

\footnotetext{
${ }^{7}$ The solution can easily be extended to the case in which two or more $\lambda_{k}$ are equal.
}

\section{PERformance EVAluation}

In this section numerical results for the CRBs are obtained and the effects of the optimal weight selection on the accuracy of TOA estimation are investigated in the absence/presence of interference. Also, the ML TOA estimation algorithm is derived and its performance is evaluated by simulation to assess the impact of the optimal weight selection on a practical estimator.

\section{A. ML TOA Estimation Algorithm}

Without loss of generality we assume that $\{1,2, \ldots, \bar{K}\}$, with $\bar{K} \leq K$, is the subset of indices $k$ corresponding to $w_{k}>0$. Then (4) can be written in the equivalent form

$$
s_{r}(t)=\sum_{k=1}^{\bar{K}} \alpha_{k} \sqrt{w_{k}} p(t) e^{\jmath 2 \pi f_{k} t} .
$$

Letting $g_{k}(t)=\sqrt{w_{k}} p(t) e^{j 2 \pi f_{k} t} \otimes h(t)$ and taking (49) into account, the log-likelihood function reads

$$
\ln \Lambda(\widetilde{\boldsymbol{\theta}})=\Re\left\{\sum_{k=1}^{\bar{K}} \tilde{\alpha}_{k}^{*} x_{k}(\tilde{\tau})\right\}-\frac{1}{2} \sum_{k=1}^{\bar{K}} \sum_{l=1}^{\bar{K}} \tilde{\alpha}_{k}^{*} \tilde{\alpha}_{l} \rho_{k, l}
$$

with

$$
x_{k}(\tilde{\tau})=\int_{-\infty}^{\infty} x(t) g_{k}^{*}(t-\tilde{\tau}) d t
$$

and

$$
\begin{aligned}
\rho_{k, l} & =\int_{-\infty}^{\infty} g_{k}^{*}(t-\tilde{\tau}) g_{l}(t-\tilde{\tau}) d t=\int_{-\infty}^{\infty} g_{k}^{*}(t) g_{l}(t) d t \\
& =\int_{-\infty}^{\infty} G_{k}^{*}(f) G_{l}(f) d f \\
& =\sqrt{w_{k}} \sqrt{w_{l}} \int_{-\infty}^{\infty} P^{*}\left(f-f_{k}\right) P\left(f-f_{l}\right)|H(f)|^{2} d f
\end{aligned}
$$

Using a matrix notation, (50) can be written as

$$
\ln \Lambda(\widetilde{\boldsymbol{\theta}})=\Re\left\{\widetilde{\boldsymbol{\alpha}}^{H} \mathbf{x}(\tilde{\tau})\right\}-\frac{1}{2} \widetilde{\boldsymbol{\alpha}}^{H} \mathbf{R} \widetilde{\boldsymbol{\alpha}}
$$

where $\widetilde{\boldsymbol{\alpha}}=\left[\tilde{\alpha}_{1}, \tilde{\alpha}_{2}, \ldots, \tilde{\alpha}_{\bar{K}}\right]^{T}, \quad \mathbf{x}(\tilde{\tau})=$ $\left[x_{1}(\tilde{\tau}), x_{2}(\tilde{\tau}), \ldots, x_{\bar{K}}(\tilde{\tau})\right]^{T}$, and $\mathbf{R}$ is the Hermitian $\bar{K} \times \bar{K}$ correlation matrix

$$
\mathbf{R}=\left[\begin{array}{cccc}
\rho_{1,1} & \rho_{1,2} & \cdots & \rho_{1, \bar{K}} \\
\rho_{2,1} & \rho_{2,2} & \cdots & \rho_{2, \bar{K}} \\
\vdots & \vdots & \ddots & \vdots \\
\rho_{\bar{K}, 1} & \rho_{\bar{K}, 2} & \cdots & \rho_{\bar{K}, \bar{K}}
\end{array}\right] .
$$

Our goal is to maximize (53) with respect to $\tilde{\tau}$ and $\widetilde{\boldsymbol{\alpha}}$. To this purpose, taking $\tilde{\tau}$ fixed and letting $\widetilde{\boldsymbol{\alpha}}$ vary, the maximum of (53) is achieved for

$$
\widehat{\boldsymbol{\alpha}}=\mathbf{R}^{-1} \mathbf{x}(\tilde{\tau})
$$

Next, substituting (55) in (53) and maximizing with respect to $\tilde{\tau}$ produces

$$
\hat{\tau}=\arg \max _{\tilde{\tau}}\left\{\mathbf{x}^{H}(\tilde{\tau}) \mathbf{R}^{-1} \mathbf{x}(\tilde{\tau})\right\} .
$$




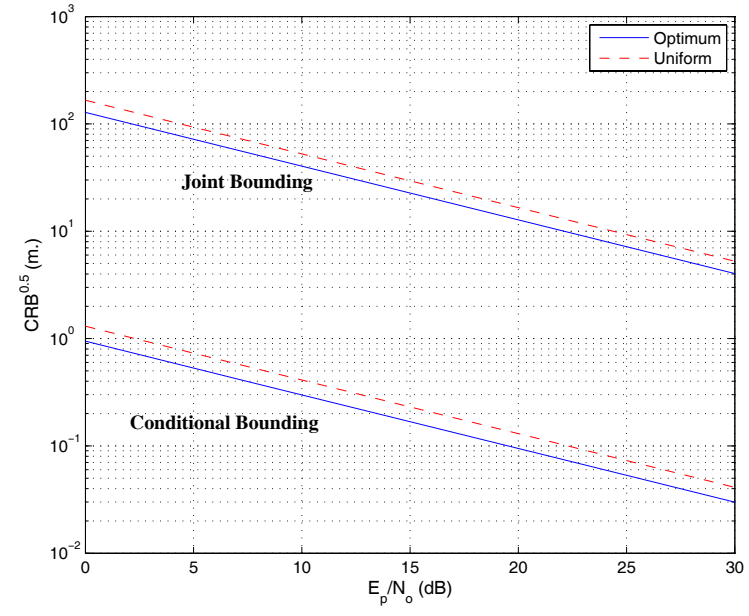

Fig. 1. $\sqrt{\mathrm{CRB}}$ versus $E_{p} / N_{0}$ for optimal and conventional (uniform) algorithm in the absence of interference.

The last equation gives the desired ML estimate of $\tau$. The ML estimate of $\alpha$ is obtained from (55) by replacing $\tilde{\tau}$ with $\hat{\tau}$ (however, we are not interested in estimating $\boldsymbol{\alpha}$ ).

From (52) it can be seen that, under the disjoint spectrum assumption made in Section III-A, we have $\rho_{k, l}=0$ for $k \neq l$. Accordingly, (56) becomes

$$
\hat{\tau}=\arg \max _{\tilde{\tau}}\left\{\sum_{k=1}^{\bar{K}} \rho_{k, k}^{-1}\left|x_{k}(\tilde{\tau})\right|^{2}\right\} .
$$

It should be noted that the time delay estimators in (56) and (57) require a simple one-dimensional search, whose complexity increases only linearly with the duration of the search interval.

\section{B. Numerical Examples}

A scenario with a subcarrier spacing $\Delta=1 \mathrm{MHz}$ and $K=128$ subcarriers is considered. The channel coefficients $\alpha_{k}$ are modeled as independent complex-valued Gaussian random variables with unit average power. The results are obtained by averaging over 500 independent channel realizations. Pulse $p(t)$ in (1) is modeled as a sinc pulse; namely, $p(t)=\sqrt{E_{p} \Delta} \sin (\pi t \Delta) /(\pi t \Delta)$. Parameters $\beta_{1}$ and $\beta_{2}$ in (33) are set to 0 and $\Delta^{2} / 12$, respectively. The results are expressed in terms of the square-root of the CRB on the ranging error, which is computed as the product of the squareroot of the CRB on TOA error multiplied by the speed of light.

In Fig. 1 the square-root of the CRB (in meters) is plotted against $E_{p} / N_{0}$ in the absence of interference for the optimal algorithm (whose weights are computed from (47)) and for the conventional algorithm that assigns equal weights to the subcarriers (uniform). It is assumed that $w_{k}$ cannot exceed $2 / K$, which implies that the power constraint defined in Section III-C is specified by $b_{k}=2 / K$ for $k=1, \ldots, K$. Both joint and conditional bounds are drawn (see Sections III-A and III-B). The figure shows that a gain of about $3 \mathrm{~dB}$ in terms of $E_{p} / N_{0}$ is obtained with the optimal weights. However, the conditional bounding gives very low (optimistic) results compared with the joint bounding for it assumes knowledge of the channel gains. Henceforth we concentrate on joint bounding.

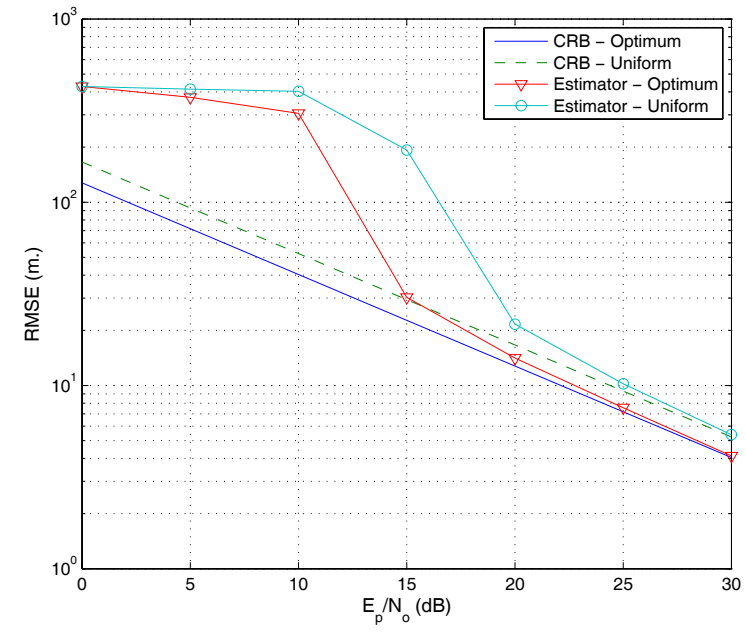

Fig. 2. RMSE versus $E_{p} / N_{0}$ for the practical TOA estimation algorithms based on optimal and uniform weight assignments. Also, the CRBs are illustrated for both cases. No interference is assumed in this scenario.

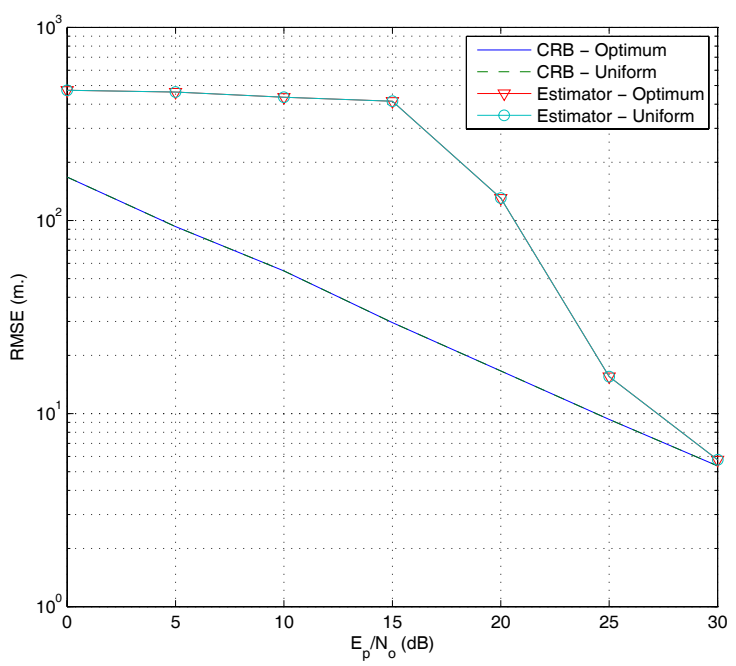

Fig. 3. RMSE versus $E_{p} / N_{0}$ for the optimal and conventional (uniform) algorithms in the presence of interference with a flat spectral density in the interval $23 \leq k \leq 106$. In this scenario, the subcarriers with interference are not used (interference avoidance).

Next, the performance of the ML TOA estimator in (57) is investigated for optimal and uniform weight assignments. The aim is to see whether the optimal assignment, which is based on the CRB minimization, is also effective in practical TOA estimators. In Fig. 2, the root-mean-squared error (RMSE) of the TOA estimator is shown with optimal and uniform weights and is compared with the corresponding CRBs. It is seen that the optimal weights also improve the performance of the TOA estimator.

Now we concentrate on the effects of interference. The system parameters are all as before. The interference spectral density $S_{I}(f)$ takes a constant value of $N_{I}=2 N_{0}$ for the subcarrier indices from 23 to 106 while it is zero elsewhere. In Fig. 3 the performance of the TOA estimator and the CRB are illustrated with optimal and conventional (uniform) weights in the case of an interference avoidance strategy. This means that the transmitted power is set to zero at the 


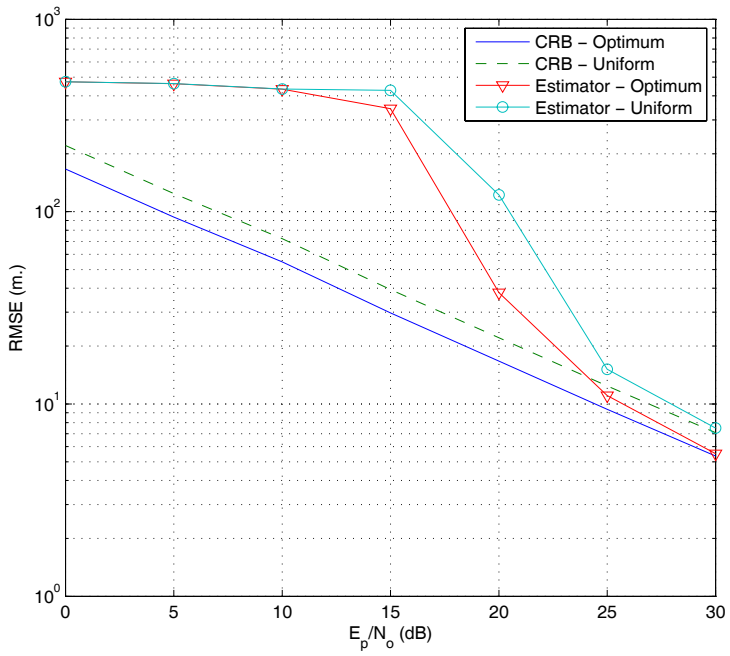

Fig. 4. RMSE versus $E_{p} / N_{0}$ for the optimal and conventional (uniform) algorithms in the presence of interference with a flat spectral density in the interval $23 \leq k \leq 106$. In this scenario, the subcarriers with interference are also used.

subcarriers with interference (i.e., $w_{k}=0$ for $23 \leq k \leq 106$ ) while uniform or optimal power allocation is used with the remaining subcarriers. Unlike Fig. 2, it is seen that the optimal and uniform allocation strategies provide the same TOA estimation accuracy in this case. In addition, it is observed that the estimation errors increase significantly in the presence of interference when the subcarriers with interference are not utilized. In Fig. 4 the same scenario is considered except that all the subcarriers can now be employed. In this case, it is observed that the optimal algorithm improves both the CRB and the TOA estimation accuracy of the ML algorithm compared to the conventional (uniform) algorithm. In addition, the mean error values are smaller than those in the interference avoidance case, as expected (see Fig. 3). ${ }^{8}$ We conclude that subcarriers with interference should be employed to better utilize the frequency diversity and enhance TOA estimation performance.

The improvement obtainable by using all the subcarriers (instead of the interference-free ones only) depends on the interference power and the number of subcarriers with interference. Specifically, the improvement reduces as the number of subcarriers with interference decreases and/or the interference power increases. Figs. 5 and 6 show the CRB and the performance of the ML TOA estimator for interference-avoidance and no-avoidance cases, respectively, when the interference spectrum extends from subcarrier 49 to subcarrier 80 with a spectral density of $N_{I}=4 N_{0}$. We see that the gain achieved by exploiting all the subcarriers is less significant compared with the scenarios discussed in Figs. 3 and 4. Still, a significant advantage is obtained with the optimal weights in place of the conventional ones.

In order to explain the mechanisms behind the results in Fig. 5 and Fig. 6, Fig. 7 illustrates a realization of the channel coefficients and the corresponding optimal weights at

${ }^{8}$ For the sake of fairness it should be noted that the transmitted signal powers (see (2)) are not the same in Figs. 4 and 5 due to the power constraint, $2 / K$. Specifically, in the former $\sum_{k=1}^{K} w_{k}$ equals $\frac{2}{128} \times 44=0.6875$ while in the latter it is unity.

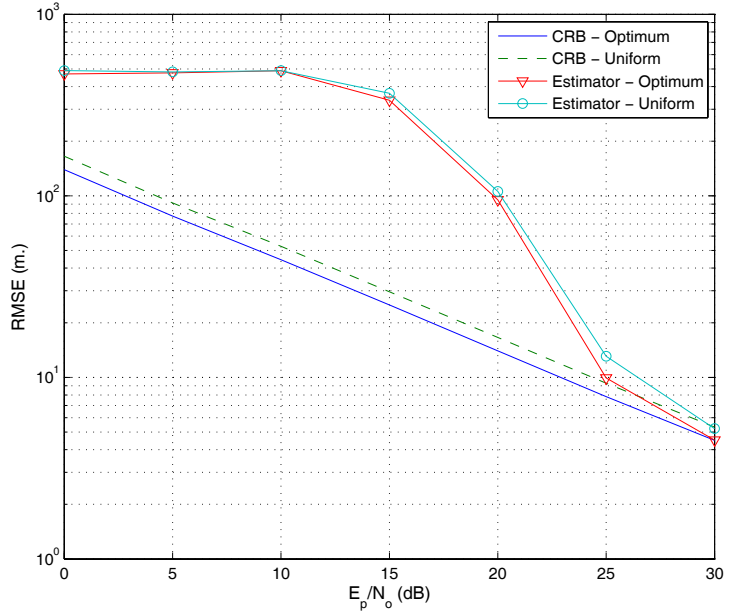

Fig. 5. RMSE versus $E_{p} / N_{0}$ for the optimal and conventional (uniform) algorithms in the presence of interference with a flat spectral density of $4 N_{0}$ in the interval $49 \leq k \leq 80$. In this scenario, the subcarriers with interference are not used (interference avoidance).

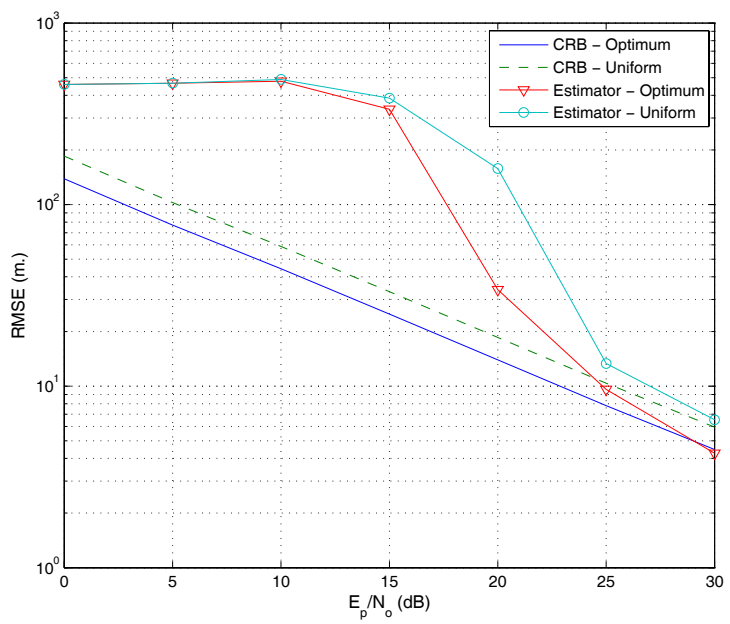

Fig. 6. RMSE versus $E_{p} / N_{0}$ for the optimal and conventional (uniform) algorithms in the presence of interference with a flat spectral density of $4 N_{0}$ in the interval $49 \leq k \leq 80$. In this scenario, the subcarriers with interference are also used.

$E_{p} / N_{0}=30 \mathrm{~dB}$ in two cases: one using only the interferencefree subcarriers (interference avoidance) (Fig. 7-(c)), the other employing all the subcarriers (Fig. 7-(d)). In agreement with (30) and (47) we see that the subcarriers with large $\lambda_{k}$ 's and/or small interference are favored in the optimal spectrum. Correspondingly, the optimal power allocation algorithm provides improved TOA estimation performance compared to the uniform algorithm, as can be seen in Fig. 6.

Fig. 8 illustrates how the power of the interference dynamically affects the CRB and the performance of the ML TOA estimator through the signal-to-interference ratio (SIR). An increase in the interference spectral density results in an increase in the RMSE of the ML TOA estimator while the corresponding CRB is not influenced significantly since only one fourth of the subcarriers experience interference. It is also observed that, as the interference power decreases, the gain from the utilization of the optimal power allocation scheme increases in both scenarios. 

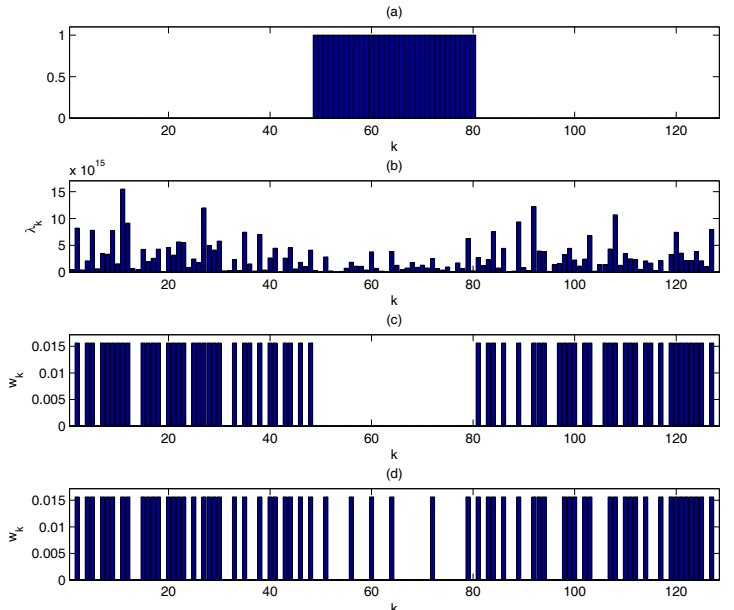

Fig. 7. (a) Spectrum of the interference. (b) Subcarrier coefficient $\lambda_{k}$ versus subcarrier index $k$. (c) Subcarrier weights versus subcarrier index for the optimal algorithm that uses only the interference-free subcarriers (interference avoidance). (d) Subcarrier weights versus subcarrier index for the optimal algorithm that uses all the subcarriers.

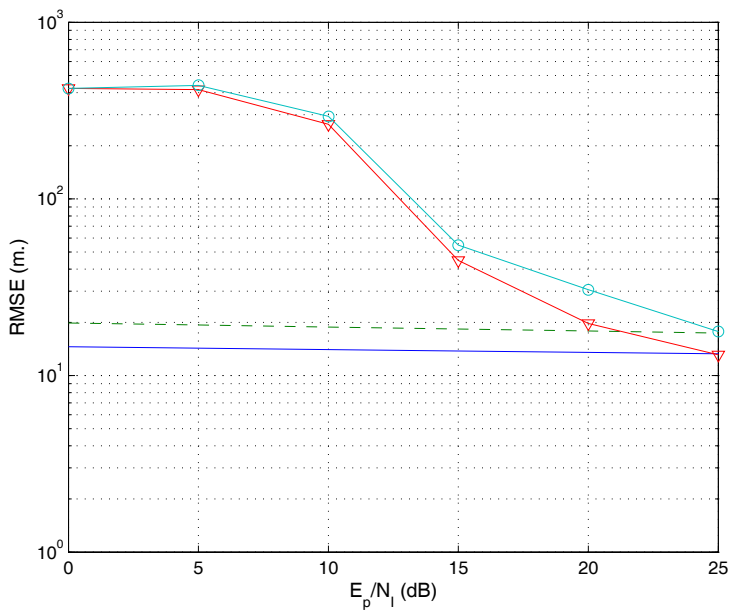

Fig. 8. RMSE versus SIR (defined as $E_{p} / N_{I}$ ) for the optimal and conventional (uniform) algorithms in the presence of interference in the interval $49 \leq k \leq 80$, where $E_{p} / N_{0}=20 \mathrm{~dB}$. In this scenario, the subcarriers with interference are also used.

Finally, the sensitivity of the CRBs and the ML estimators to spectral estimation errors is investigated. It is assumed that interference spectral density $S_{I}(f)$ takes a constant value of $N_{I}=4 N_{0}$ for the subcarrier indices from 23 to 106 while it is zero elsewhere. Assuming that the spectral estimation error can be modeled as a zero-mean Gaussian random variable with variance $\sigma_{e}^{2}$, Table I presents the RMSE values of the ML estimators and the CRBs for the optimal and uniform power allocation strategies for various spectral estimation error variances at $E_{p} / N_{0}=30 \mathrm{~dB}$ for the scenario in which all the subcarriers are used. It is observed that an increase in the uncertainty of spectral estimation (that is, $\sigma_{e}^{2}$ ) leads to an increase in the CRB and the RMSE of the ML TOA estimator for the optimal power allocation strategy. On the other hand, when the uniform power allocation strategy is used, the performance is not affected by the spectral esti-
TABLE I

RMSE (IN METERS) VS. SPECTRUM ESTIMATION ERROR VARIANCE, $\sigma_{e}^{2}$

\begin{tabular}{|c|c|c|c|c|}
\hline & $\sigma_{e}^{2}=0$ & $\sigma_{e}^{2}=0.5$ & $\sigma_{e}^{2}=1$ & $\sigma_{e}^{2}=1.5$ \\
\hline Estimator - Optimal & 8.402 & 8.565 & 8.622 & 8.720 \\
\hline Estimator - Uniform & 10.58 & 10.58 & 10.58 & 10.58 \\
\hline$\sqrt{\mathrm{CRB}}-$ Optimal & 5.611 & 5.681 & 5.718 & 5.750 \\
\hline$\sqrt{\mathrm{CRB}}-$ Uniform & 7.617 & 7.617 & 7.617 & 7.617 \\
\hline
\end{tabular}

mation errors. The reason for this is that the optimal power allocation strategy uses the knowledge of the interference level whereas the uniform one always assigns equal powers to all the subcarriers irrespective of the interference level. Although the optimal power allocation strategy is affected by the spectral estimation errors, it is also noted that its performance is consistently superior to that of the uniform power allocation strategy even for substantially high values of spectral estimation errors. This demonstrates the robustness of the optimal power allocation scheme against uncertainties in the spectral estimation mechanism of the system.

\section{CONCLUSIONS}

Theoretical limits on TOA estimation have been studied for multicarrier systems in the presence of interference. Specifically, closed form CRB expressions have been obtained for TOA estimation. Based on these expressions an optimal signal power allocation strategy has been proposed, which considers both the interference spectrum and the regulatory emission mask. The CRB and the performance of the ML TOA estimator have been investigated via numerical examples. It has been observed that the intuitive interference avoidance strategy, which assigns signal power only to the interferencefree subcarriers, is not optimal. In other words, the frequency diversity can be utilized more efficiently if all the subcarriers, including the ones with interference, are employed for TOA estimation. The results provide guidelines for time delay estimation in multicarrier systems, such as 3GPP-LTE and IEEE 802.16 WiMAX.

\section{APPENDIX}

\section{A. Derivation of (7)}

The likelihood function at the output of the whitening filter $h(t)$ can be written as [32]

$$
\bar{\Lambda}(\widetilde{\boldsymbol{\theta}})=\exp \left\{-\int_{-\infty}^{\infty}|x(t)-u(t, \widetilde{\boldsymbol{\theta}})|^{2} \mathrm{~d} t\right\}
$$

where the interference plus noise power spectral density $S_{z}(f)$ is taken to be unity since the total disturbance $z(t)$ is already whitened. Expanding the term inside the curly braces, we get

$$
\begin{array}{r}
\bar{\Lambda}(\widetilde{\boldsymbol{\theta}})=\exp \left\{-\int_{-\infty}^{\infty}|x(t)|^{2} \mathrm{~d} t+2 \Re\left\{\int_{-\infty}^{\infty} x(t) u^{*}(t, \widetilde{\boldsymbol{\theta}})\right\}\right. \\
\left.-\int_{-\infty}^{\infty}|u(t, \widetilde{\boldsymbol{\theta}})|^{2} \mathrm{~d} t\right\} .
\end{array}
$$

Note that the first term inside the exponent of (59) does not involve the channel parameter vector $\widetilde{\boldsymbol{\theta}}=$

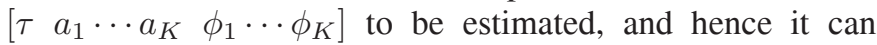


be neglected in finding the estimate $\hat{\boldsymbol{\theta}}$ that maximizes the likelihood function. Dividing the rest of the exponent by 2 yields the following equivalent likelihood function

$\Lambda(\widetilde{\boldsymbol{\theta}})=\exp \left\{\Re\left\{\int_{-\infty}^{\infty} x(t) u^{*}(t, \widetilde{\boldsymbol{\theta}}) \mathrm{d} t\right\}-\frac{1}{2} \int_{-\infty}^{\infty}|u(t, \widetilde{\boldsymbol{\theta}})|^{2} \mathrm{~d} t\right\}$

since taking the square root of an always positive objective function has no effect on the maximizing parameter value. From (60), the corresponding log-likelihood function can be written as

$$
\ln \Lambda(\widetilde{\boldsymbol{\theta}})=\Re\left\{\int_{-\infty}^{\infty} x(t) u^{*}(t, \widetilde{\boldsymbol{\theta}}) \mathrm{d} t\right\}-\frac{1}{2} \int_{-\infty}^{\infty}|u(t, \widetilde{\boldsymbol{\theta}})|^{2} \mathrm{~d} t .
$$

\section{B. Derivation of (10)}

Substitution of

$$
x(t)=r(t) \otimes h(t)=\int_{-\infty}^{\infty} r(z) h(t-z) \mathrm{d} z
$$

and

$$
u(t, \widetilde{\boldsymbol{\theta}})=\tilde{s}_{r}(t-\tilde{\tau}) \otimes h(t)=\int_{-\infty}^{\infty} \tilde{s}_{r}(z-\tilde{\tau}) h(t-z) \mathrm{d} z
$$

into the log-likelihood function in (7) gives

$$
\begin{aligned}
& \ln \Lambda(\widetilde{\boldsymbol{\theta}})= \\
& \Re\left\{\int_{-\infty}^{\infty} \int_{-\infty}^{\infty} r(z) h(t-z) \mathrm{d} z \int_{-\infty}^{\infty} \tilde{s}_{r}^{*}(v-\tilde{\tau}) h^{*}(t-v) \mathrm{d} v \mathrm{~d} t\right\} \\
& -\left\{\frac{1}{2} \int_{-\infty}^{\infty} \int_{-\infty}^{\infty} \tilde{s}_{r}(z-\tilde{\tau}) h(t-z) \mathrm{d} z\right. \\
& \left.\int_{-\infty}^{\infty} \tilde{s}_{r}^{*}(v-\tilde{\tau}) h^{*}(t-v) \mathrm{d} v \mathrm{~d} t\right\}
\end{aligned}
$$

Defining a new function

$$
\begin{aligned}
Q(z, v) & \triangleq \int_{-\infty}^{\infty} h(t-z) h^{*}(t-v) \mathrm{d} t \\
& =\int_{-\infty}^{\infty} h(t) h^{*}(t-v+z) \mathrm{d} t
\end{aligned}
$$

leads to the following expression for the log-likelihood function:

$$
\begin{array}{r}
\ln \Lambda(\widetilde{\boldsymbol{\theta}})=\Re\left\{\int_{-\infty}^{\infty} r(z) \int_{-\infty}^{\infty} Q(z, v) \tilde{s}_{r}^{*}(v-\tilde{\tau}) \mathrm{d} v \mathrm{~d} z\right\} \\
-\left\{\frac{1}{2} \int_{-\infty}^{\infty} \tilde{s}_{r}(z-\tilde{\tau}) \int_{-\infty}^{\infty} Q(z, v) \tilde{s}_{r}^{*}(v-\tilde{\tau}) \mathrm{d} v \mathrm{~d} z\right\} .
\end{array}
$$

The expression in (66) can be further simplified by defining

$$
g(t, \widetilde{\boldsymbol{\theta}}) \triangleq \int_{-\infty}^{\infty} Q^{*}(t, v) \tilde{s}_{r}(v-\tilde{\tau}) \mathrm{d} v
$$

which results in the same expression for log-likelihood function as in (10); i.e.,

$$
\begin{aligned}
\ln \Lambda(\widetilde{\boldsymbol{\theta}}) & =\Re\left\{\int_{-\infty}^{\infty} r(t) g^{*}(t, \widetilde{\boldsymbol{\theta}}) \mathrm{d} t\right\} \\
& -\frac{1}{2} \int_{-\infty}^{\infty} \tilde{s}_{r}(t-\tilde{\tau}) g^{*}(t, \widetilde{\boldsymbol{\theta}}) \mathrm{d} t .
\end{aligned}
$$

The Fourier transform $G(f, \widetilde{\boldsymbol{\theta}})$ of $g(t, \widetilde{\boldsymbol{\theta}})$ can be computed as

$$
\begin{aligned}
G(f, \widetilde{\boldsymbol{\theta}})=\int_{-\infty}^{\infty} g(t, \widetilde{\boldsymbol{\theta}}) e^{-\jmath 2 \pi f t} \mathrm{~d} t \\
=\int_{-\infty}^{\infty} \tilde{s}_{r}(v-\tilde{\tau}) \int_{-\infty}^{\infty} Q^{*}(t, v) e^{-\jmath 2 \pi f t} \mathrm{~d} t \mathrm{~d} v \\
=\int_{-\infty}^{\infty} \tilde{s}_{r}(v-\tilde{\tau}) \int_{-\infty}^{\infty} h^{*}(u) \int_{-\infty}^{\infty} h(u-v+t) e^{-\jmath 2 \pi f t} \mathrm{~d} t \mathrm{~d} u \mathrm{~d} v \\
=\int_{-\infty}^{\infty} \tilde{s}_{r}(v-\tilde{\tau}) e^{-\jmath 2 \pi f v} \int_{-\infty}^{\infty} h^{*}(u) e^{\jmath 2 \pi f u}
\end{aligned}
$$$$
\int_{-\infty}^{\infty} h(u-v+t) e^{-\jmath 2 \pi f(t+u-v)} \mathrm{d} t \mathrm{~d} u \mathrm{~d} v
$$$$
=\int_{-\infty}^{\infty} h(t) e^{-\jmath 2 \pi f t} \mathrm{~d} t \int_{-\infty}^{\infty} h^{*}(u) e^{\jmath 2 \pi f u} \mathrm{~d} u
$$$$
\int_{-\infty}^{\infty} \tilde{s}_{r}(v-\tilde{\tau}) e^{-\jmath 2 \pi f v} \mathrm{~d} v
$$$$
=|H(f)|^{2} \tilde{S}_{r}(f, \widetilde{\boldsymbol{\theta}})=\frac{\tilde{S}_{r}(f, \widetilde{\boldsymbol{\theta}})}{S_{z}(f)}
$$

where $\tilde{S}_{r}(f, \widetilde{\boldsymbol{\theta}})$ is the Fourier transform of $\tilde{s}_{r}(t-\tilde{\tau})$ in (8), and the final expression in (69) proves (9).

\section{Derivation of (47)}

In this appendix we show that (48) is the solution of the maximization problem (43). The proof is recursive, i.e., we first show that $w_{1}^{(\mathrm{opt})}=\min \left\{1, b_{1}\right\}$, then $w_{2}^{(\mathrm{opt})}=\min \{1-$ $\left.w_{1}^{(\mathrm{opt})}, b_{2}\right\}$, next $w_{3}^{(\mathrm{opt})}=\min \left\{1-w_{1}^{(\mathrm{opt})}-w_{2}^{(\mathrm{opt})}, b_{3}\right\}$ and so on.

We begin with proving that $w_{1}^{(\mathrm{opt})}=\min \left\{1, b_{1}\right\}$. As a first step in this direction we observe that $w_{1}^{\text {(opt) }}$ must be smaller than or equal to $\min \left\{1, b_{1}\right\}$. This is so because (44)(45) require $w_{1}^{(\mathrm{opt})} \leq 1$, while (46) requires $w_{1}^{(\mathrm{opt})} \leq b_{1}$. Thus, it must be:

$$
w_{1}^{(\mathrm{opt})}=\min \left\{1, b_{1}\right\}-\delta, \quad \delta \geq 0 .
$$

We maintain that assuming $\delta>0$ leads to a contradiction. In fact, we show that, if the optimal solution $\boldsymbol{w}^{(\mathrm{opt})}=$ 
$\left(w_{1}^{(\mathrm{opt})}, w_{2}^{(\mathrm{opt})}, \ldots, w_{K}^{(\mathrm{opt})}\right)^{T}$ has the first component smaller than $\min \left\{1, b_{1}\right\}$, then a vector $\boldsymbol{w}$ can be found that satisfies (44)-(46) and has a scalar product $\boldsymbol{\lambda}^{T} \boldsymbol{w}$ greater than $\lambda^{T} \boldsymbol{w}^{(\mathrm{opt})}$.

To proceed, we distinguish two cases, say $(a)$ and $(b)$, according to whether $\delta$ is smaller or greater than $\sum_{k=2}^{n} w_{k}^{(\mathrm{opt})}$.

1) Case (a): Assume $0<\delta \leq \sum_{k=2}^{n} w_{k}^{(\mathrm{opt})}$ and introduce the parameter

$$
\alpha \triangleq \delta\left(\sum_{k=2}^{n} w_{k}^{(\mathrm{opt})}\right)^{-1} .
$$

Note that $0<\alpha \leq 1$. Now consider a vector $\boldsymbol{w}$ such that

$$
w_{1}=\min \left\{1, b_{1}\right\}
$$

and

$$
w_{k}=(1-\alpha) w_{k}^{(\mathrm{opt})}, \quad k=2,3, \ldots, K .
$$

Such a vector satisfies (45) because its components are all non-negative. It also satisfies (46) because $w_{1} \leq b_{1}$ (see (72)) and $w_{k}<w_{k}^{(\mathrm{opt})} \leq b_{k}(k=2,3, \ldots, K)$ as a consequence of (73). Finally, it satisfies (44) because from (72)-(73) it is found that $\mathbf{1}^{T} \boldsymbol{w}=\mathbf{1}^{T} \boldsymbol{w}^{(\mathrm{opt})} \leq 1$.

Next consider the scalar product $\boldsymbol{\lambda}^{T} \boldsymbol{w}$. We have

$$
\begin{aligned}
\boldsymbol{\lambda}^{T} \boldsymbol{w} & =\lambda_{1} \min \left\{1, b_{1}\right\}+\sum_{k=2}^{K} \lambda_{k} w_{k} \\
& =\lambda_{1} \min \left\{1, b_{1}\right\}+\sum_{k=2}^{K} \lambda_{k} w_{k}^{(\mathrm{opt})}-\alpha \sum_{k=2}^{K} \lambda_{k} w_{k}^{(\mathrm{opt})} \\
& >\lambda_{1} \min \left\{1, b_{1}\right\}+\sum_{k=2}^{K} \lambda_{k} w_{k}^{(\mathrm{opt})}-\alpha \lambda_{1} \sum_{k=2}^{K} w_{k}^{(\mathrm{opt})} \\
& =\lambda_{1} \min \left\{1, b_{1}\right\}+\sum_{k=2}^{K} \lambda_{k} w_{k}^{(\mathrm{opt})}-\lambda_{1} \delta \\
& =\boldsymbol{\lambda}^{T} \boldsymbol{w}^{(\mathrm{opt})}
\end{aligned}
$$

where the passage from the first to the second line follows from the fact that $\lambda_{1}$ is the largest $\lambda_{k}$, while the passage from the second to the third line is a consequence of (71). Equation (74) indicates a contradiction since $\boldsymbol{\lambda}^{T} \boldsymbol{w}^{(\mathrm{opt})}$ is the maximum possible value of $\boldsymbol{\lambda}^{T} \boldsymbol{w}$.

2) Case (b): Suppose

$$
\delta>\sum_{k=2}^{n} w_{k}^{(\mathrm{opt})}
$$

and consider a vector $\boldsymbol{w}$ such that $w_{1}$ is as in (72) while

$$
w_{k} \triangleq 0, \quad k=2,3, \ldots, K .
$$

As in the previous case, it is easily checked that $\boldsymbol{w}$ satisfies conditions (44)-(46). On the other hand, we have

$$
\boldsymbol{\lambda}^{T} \boldsymbol{w}=\lambda_{1} \min \left\{1, b_{1}\right\}
$$

while

$$
\begin{aligned}
\boldsymbol{\lambda}^{T} \boldsymbol{w}^{(\mathrm{opt})} & =\lambda_{1} \min \left\{1, b_{1}\right\}-\lambda_{1} \delta+\sum_{k=2}^{K} \lambda_{k} w_{k}^{(\mathrm{opt})} \\
& <\lambda_{1} \min \left\{1, b_{1}\right\}-\lambda_{1} \delta+\lambda_{1} \sum_{k=2}^{K} w_{k}^{(\mathrm{opt})} \\
& =\lambda_{1} \min \left\{1, b_{1}\right\}-\lambda_{1}\left(\delta-\sum_{k=2}^{K} w_{k}^{(\mathrm{opt})}\right) \\
& <\boldsymbol{\lambda}^{T} \boldsymbol{w}
\end{aligned}
$$

where the passage from the first to the second line follows from the fact that $\lambda_{1}$ is the largest $\lambda_{k}$, while the passage from the third to the fourth line is a consequence of (75) and (77). Again we see that $\boldsymbol{\lambda}^{T} \boldsymbol{w}>\boldsymbol{\lambda}^{T} \boldsymbol{w}^{(\mathrm{opt})}$, which is a contradiction. Putting cases $(a)$ and $(b)$ together, we conclude that it must be $w_{1}^{\text {(opt) }}=\min \left\{1, b_{1}\right\}$.

Next we look for the remaining components of $\boldsymbol{w}^{(\mathrm{opt})}$. Defining the $(K-1)$-dimensional vectors $\lambda^{\prime} \triangleq\left[\lambda_{2} \cdots \lambda_{K}\right]^{T}$, $\boldsymbol{b}^{\prime} \triangleq\left[b_{2} \cdots b_{K}\right]^{T}$ and $\boldsymbol{w}^{\prime} \triangleq\left[w_{2} \cdots w_{K}\right]^{T}$, our task is to solve the following problem:

$$
\underset{w}{\operatorname{maximize}} \lambda^{\prime T} \boldsymbol{w}^{\prime}
$$

subject to $\mathbf{1}^{T} \boldsymbol{w}^{\prime} \leq 1-w_{1}^{(\mathrm{opt})}, \boldsymbol{w}^{\prime} \succeq \mathbf{0}$, and $\boldsymbol{w}^{\prime} \preceq \boldsymbol{b}^{\prime}$. Reasoning as before, the first component of the optimal solution is found to be $\min \left\{1-w_{1}^{(\mathrm{opt})}, b_{2}\right\}$. Thus, we have $w_{2}^{(\mathrm{opt})}=\min \left\{1-w_{1}^{(\mathrm{opt})}, b_{2}\right\}$. Proceeding in this way, with the other components of $\boldsymbol{w}^{(\mathrm{opt})}$, produces the full solution in (47).

\section{REFERENCES}

[1] J. J. Caffery, Wireless Location in CDMA Cellular Radio Systems. Kluwer, 2000

[2] D. Dardari, E. Falletti, and M. Luise, Satellite and Terrestrial Radio Positioning Techniques - A Signal Processing Perspective. Elsevier, 2011

[3] "IEEE 15-03-0489-03-004a-application-requirement-analysis-031127 v0.4." Available: http://www.ieee802.org/15/pub/TG4.html

[4] Z. Sahinoglu, S. Gezici, and I. Guvenc, Ultra-Wideband Positioning Systems. Cambridge University Press, 2008.

[5] S. Gezici, "A survey on wireless position estimation," Wireless Personal Commun., vol. 44, no. 3, pp. 263-282, Feb. 2008.

[6] Y. Qi and H. Kobayashi, "On relation among time delay and signal strength based geolocation methods," in Proc. IEEE Global Commun. Conf., Dec. 2003, vol. 7, pp. 4079-4083.

[7] Y. Qi, H. Kobayashi, and H. Suda, "On time-of-arrival positioning in a multipath environment," IEEE Trans. Veh. Technol., vol. 55, no. 5, pp. 1516-1526, Sep. 2006.

[8] D. Dardari, A. Conti, U. Ferner, A. Giorgetti, and M. Z. Win, "Ranging with ultrawide bandwidth signals in multipath environments," Proc. IEEE, vol. 97, no. 2, pp. 404-426, Feb. 2009.

[9] M. Z. Win, A. Conti, S. Mazuelas, Y. Shen, W. M. Gifford, D. Dardari, and M. Chiani, "Network localization and navigation via cooperation," IEEE Commun. Mag., pp, 56-62, May 2011.

[10] Y. Shen, H. Wymeersch, and M. Z. Win, "Fundamental limits of wideband localization-part II: cooperative networks," IEEE Trans. Inf. Theory, vol. 56, no. 10, pp. 4981-5000, Oct. 2010.

[11] A. R. S. Bahai, B. R. Saltzberg, and M. Ergen, Multi-carrier Digital Communications: Theory and Applications Of OFDM, 2nd edition. Springer, 2004.

[12] 3GPP-LTE. Available: http://www.3gpp.org/LTE

[13] IEEE Standard for Local and Metropolitan Area Networks, "Part 16: Air interface for fixed broadband wireless access systems," IEEE Std 802.16-2004, Oct. 2004.

[14] D. Wang and M. Fattouche, "OFDM transmission for time-based range estimation," IEEE Signal Process. Lett., vol. 17, no. 6, pp. 571-574, June 2010 . 
[15] F. Zhao, W. Yao, C. C. Logothetis, and Y. Song, "Super-resolution TOA estimation in OFDM systems for indoor environments," in Proc. IEEE International Conf. Netw., Sensing Control, Apr. 2007, pp. 723-728.

[16] X. Li, K. Pahlavan, M. Latva-Aho, and M. Ylianttila, "Indoor geolocation using OFDM signals in HIPERLAN/2 wireless LANs," in Proc. 11th IEEE International Symp. Personal, Indoor Mobile Radio Commun., Sep. 2000, vol. 2, pp. 1449-1453.

[17] C. Mensing and A. Dammann, "Positioning with OFDM based communications systems and GNSS in critical scenarios," in Proc. 5th Workshop Positioning, Navigation Commun., Mar. 2008, pp. 1-7.

[18] S. Gezici, H. Celebi, H. V. Poor, and H. Arslan, "Fundamental limits on time delay estimation in dispersed spectrum cognitive radio systems," IEEE Trans. Wireless Commun., vol. 8, no. 1, pp. 78-83, Jan. 2009.

[19] F. Kocak, H. Celebi, S. Gezici, K. A. Qaraqe, H. Arslan, and H. V. Poor, "Time-delay estimation in dispersed spectrum cognitive radio systems," EURASIP J. Adv. Signal Process., vol. 2010, article ID 675959, 10 pages.

[20] S. Gezici and Z. Sahinoglu, "Ranging in a single-input multiple-output (SIMO) system," IEEE Commun. Lett., vol. 12, no. 3, pp. 197-199, Mar. 2008.

[21] D. Dardari, "Ordered subcarrier selection algorithm for OFDM based high-speed WLANs," IEEE Trans. Wireless Commun., vol. 3, no. 5, pp. 1452-1458, Sep. 2004

[22] J. O. Neel, "Analysis and design of cognitive radio networks and distributed radio resource management algorithms," Ph.D. dissertation, Virginia Polytechnic Institute and State University, VA, Sep. 2006.

[23] H. Celebi and H. Arslan, "Enabling location and environment awareness in cognitive radios," Elsevier Comput. Commun., vol. 31, no. 6, pp. 1114-1125, Apr. 2008.

[24] H. Arslan (editor), Cognitive Radio, Software Defined Radio, and Adaptive Wireless Systems. Springer, 2007.

[25] S. Gault, W. Hachem, and P. Ciblat, "Joint sampling clock offset and channel estimation for OFDM signals: Cramer-Rao bound and algorithms," IEEE Trans. Signal Process., vol. 54, no. 5, pp. 18751885, May 2006.

[26] Z. J. Wang, Z. Han, and K. J. R. Liu, "A MIMO-OFDM channel estimation approach using time of arrivals," IEEE Trans. Wireless Commun., vol. 4, no. 3, pp. 1207-1213, May 2005.

[27] A. Goldsmith, Wireless Communications. Cambridge University Press, 2005.

[28] H. Celebi and H. Arslan, "Cognitive positioning systems," IEEE Trans. Wireless Commun., vol. 6, no. 12, pp. 4475-4483, Dec. 2007.

[29] J. Mitola and G. Q. Maguire, "Cognitive radio: making software radios more personal," IEEE Personal Commun. Mag., vol. 6, pp. 13-18, Aug. 1999.

[30] B. Z. Bobrovsky, E. Mayer-Wolf, and M. Zakai, "Some classes of global Cramér-Rao bounds," Annals Statistics, vol. 15, no. 4, pp. 14211438, 1987.

[31] H. L. Van Trees, Detection, Estimation, and Modulation Theory, 1st edition. John Wiley \& Sons, Inc., 1968.

[32] J. Proakis and M. Salehi, Digital Communications. McGraw-Hill, 2007.

[33] S. K. Kay, Fundamentals of Statistical Signal Processing: Estimation Theory. Prentice-Hall, 1993.

[34] C. Snow, L. Lampe, and R. Schober, "Impact of WiMAX interference on MB-OFDM UWB systems: analysis and mitigation," IEEE Trans. Commun., vol. 57, no. 9, pp. 2818-2827, Sep. 2009.

[35] Federal Communications Commission, First Report and Order 02-48, Feb. 2002.

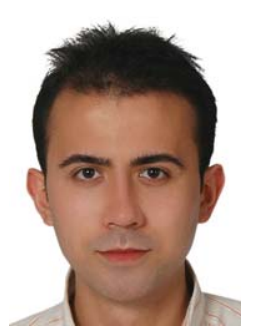

Yasir Karisan (S'09) received the B.S. degree from Koc University, Turkey, in 2008, and the M.S. degree from Bilkent University, Turkey, in 2010. He is currently working toward the Ph.D. degree in the Department of Electrical and Computer Engineering, The Ohio State University. His main research interests are in the fields of statistical signal processing and wireless communications.

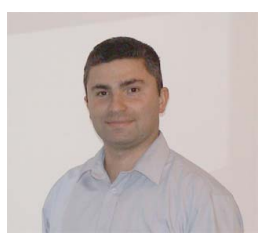

Davide Dardari (Senior Member, IEEE) received the Laurea degree in electronic engineering (summa cum laude) and the Ph.D. degree in electronic engineering and computer science from the University of Bologna, Italy, in 1993 and 1998, respectively. Currently, he is Associate Professor at the University of Bologna at Cesena, Cesena (FC), Italy. He is Research Affiliate with WiLAB and Wireless Communications Group at LIDS, Massachusetts Institute of Technology (MIT). His actual research interests are in ultrawide bandwidth communication and localization, wireless sensor networks, OFDM systems. He published more than 100 technical papers and played several important roles in various National and European Projects. Davide Dardari is the current Chair of the Radio Communications Committee of the IEEE Communications Society. He is cogeneral Chair of the IEEE International Conference on UWB (ICUWB) 2011. He was co-chair of the Wireless Communications Symposium of the 2007 IEEE International Conference on Communications (ICC) and of the IEEE ICUWB 2006. He was Lead Editor for the EURASIP Journal on Advances in Signal Processing (Special Issue on Cooperative Localization in Wireless Ad Hoc and Sensor Networks), Guest Editor for ProceEdings of THE IEEE (Special Issue on UWB Technology \& Emerging Applications), Guest Editor for Physical Communication Journal (ELSEVIER) (Special Issue on Advances in Ultra-Wideband Wireless Communications), and Guest Editor for EURASIP (Hindawi) Journal on Wireless Communications and Networking (Special Issue on Signal Processing-assisted Protocols and Algorithms for Cooperating Objects and Wireless Sensor Networks). Currently, he is an Associate Editor for IEEE TRANS ACTIONS ON WIRELESS COMMUNICATIONS.

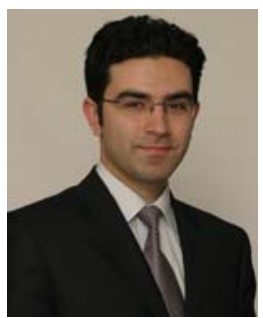

Sinan Gezici (S'02, M'06, SM'11) received the B.S. degree from Bilkent University, Turkey in 2001, and the Ph.D. degree in Electrical Engineering from Princeton University in 2006. From 2006 to 2007, he worked at Mitsubishi Electric Research Laboratories, Cambridge, MA. Since February 2007, he has been an Assistant Professor in the Department of Electrical and Electronics Engineering at Bilkent University.

Dr. Gezici's research interests are in the areas of signal detection, estimation and optimization theory, and their applications to wireless communications and localization systems. Among his publications in these areas is the book Ultra-Wideband Positioning Systems: Theoretical Limits, Ranging Algorithms, and Protocols (Cambridge University Press, 2008).

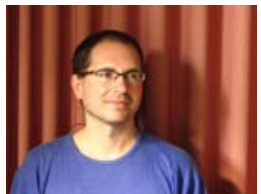

Antonio A. D'Amico received the Dr. Ing. Degree in Electronic Engineering in 1992 and the Ph.D. degree in 1997, both from the University of Pisa, Italy. He is currently a Research Fellow at the Department of Information Engineering of the University of Pisa. His research interests are in digital communication theory, with emphasis on synchronization algorithms, channel estimation and detection techniques.

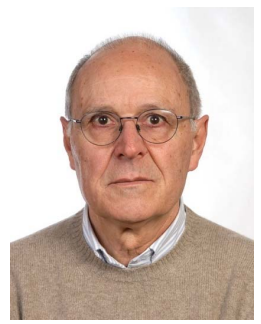

Umberto Mengali is Professor Emeritus of Telecommunications in the University of Pisa. His research interests are in the area of wireless communications and ultra-wideband systems, with emphasis on synchronization methods and modulation techniques. He has published about a hundred journal papers and has co-authored the book Synchronization Techniques for Digital Receivers (Plenum Press). Professor Mengali is Life Fellow of the IEEE. 